\title{
Effect of tooth brushing with a novel dentifrice on porcelain roughness and gloss
}

\author{
Abstract \\ Objectives: The objective of this study was to evaluate the effect of a novel dentifrice \\ (Diamynt, with fluoride, xylitol, herbal extracts, hydrated silica and diamond particles) on \\ the surface roughness and gloss of two commercially available dental porcelains following \\ simulated toothbrushing.
}

Methods: Two commercially available porcelains (Vita VM13 and Ceramco 3) were used, as well as commercial finishing and polishing instruments (Dialite wheels). The porcelain samples were fabricated and polished by a professional dental laboratory with the same dimensions and polishing method. Roughness ( $\mathrm{Ra}$ ) was evaluated with a stylus profilometer (Federal PocketSurf). Gloss measurements were obtained by using a $60^{\circ}$ small object glossmeter (Rhopoint NovoCurve Glossmeter). Following initial surface analysis of gloss and profilometry, the polished side of every sample was treated with a simulated toothbrushing technique previously published using a toothbrushing device (Sabri Dental V-8 Toothbrush Abrasion Machine). Diamynt and Arm \& Hammer Whitening toothpaste were independently used to create a 50:50 w/w slurry with deionized water. Each surface was brushed 10,000 times. (Equivalent to 1 year toothbrushing) Then, the samples were rinsed with tap water and stored in $100 \%$ humidity until roughness and gloss values were obtained using the glossmeter and profilometer. Data was analyzed using two-tailed, unequal variance t-tests.

Results: Diamynt toothpaste had no significant effect on surface roughness or gloss on either porcelain tested. Arm and Hammer had no effect on the surface roughness of either porcelain, but a significant effect $(\mathrm{P}<0.05)$ on surface gloss of Ceramco 3 porcelain.

Conclusion: Effects of toothbrush abrasion was shown to be material and toothpaste dependent.
Volume I Issue 3 - 2014

\author{
Garcia Godoy, RA Wicks, F Garcia Godoy, B \\ Morrow, Lisa Marie Kao \\ College of Dentistry and College of Medicine, University of \\ Tennessee Health Science Center, USA
}

\begin{abstract}
Correspondence: Garcia-Godoy, College of Dentistry and College of Medicine, University of Tennessee Health Science Center (UTHSC), 920 Madison Ave, Memphis, TN 38163, USA,
\end{abstract} Email agodoy2747@gmail.com

Received: June 13,2014 | Published: June 18, 2014

\section{Introduction}

The size of the filler particles that are incorporated into a dentifrice can affect the color properties and surface roughness of both natural teeth and prosthetic surfaces. Traditional measurement of color relies on evaluation of hue, chroma, value, but a fourth factor is gloss. Gloss can be measured in a relative unit named "Gloss units," a scale from 0 (matte, no reflectance) to 100 (polished, mirror-like surface). Roughness, measured in Ra serves as an indicator of how abrasivity has affected a sample after being repeatedly passed over a rough surface. Due to their common use as veneering materials over metal substructures for porcelain fused-to-metal crowns, Vita Zahnfabrik VM 13 and Dentsply Ceramco 3 porcelain were chosen as suitable determinant surfaces for abrasion with dentifrices (Chart 1).

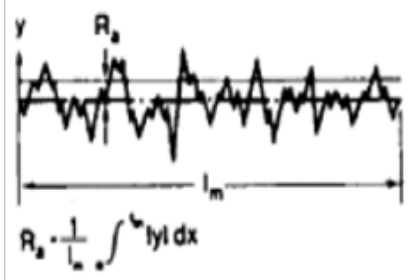

\[ \text { The } R_{a} \text {-value as a } \]
function of surface profile. $R_{d}$ is
defined as the arithmetical mean
value of all distances of the pro-
file $R$ to the mean distance within
the length $l_{m}$

Chart I

\section{Purpose}

\section{Objectives}

The objective of this study was to evaluate the effect of a novel dentifrice (Diamynt, with fluoride, xylitol, herbal extracts, hydrated silica and diamond particles) on the surface roughness and gloss of two commercially available dental porcelains following simulated toothbrushing.

\section{Material and methods}

Two commercially available porcelains (Vita VM13 and Ceramco 3) were used, as well as commercial finishing and polishing instruments (Dialite wheels). Sixteen samples were fabricated for each group. The porcelain samples were fabricated and polished by a professional dental laboratory with the same dimensions and polishing method. Roughness (Ra) was evaluated with a stylus profilometer (Federal PocketSurf). Gloss measurements were obtained by using a $60^{\circ}$ small object glossmeter (Rhopoint NovoCurve Glossmeter). Following initial surface analysis of gloss and profilometry, the polished side of every sample was treated with a simulated toothbrushing technique previously published1 using a toothbrushing device (Sabri Dental V-8 Toothbrush Abrasion Machine). Diamynt and Arm \& Hammer Whitening toothpaste were independently used to create $50: 50 \mathrm{w} / \mathrm{w}$ slurry with deionized water. Each surface was brushed 10,000 times. (Equivalent to 1 year toothbrushing) Then, the samples were rinsed 
with tap water and stored in 100\% humidity until roughness and gloss values were obtained using the glossmeter and profilometer. Data was analyzed using two-tailed, unequal variance t-tests.

\section{Results}

Diamynt toothpaste had no significant effect on surface roughness or gloss on either porcelain tested. Arm and Hammer had no effect on the surface roughness of either porcelain, but a significant effect $(\mathrm{P}<0.05)$ on surface gloss of Ceramco 3 porcelain. Data here represents four test groups (Vita and Ceramco/Diamynt and Arm \& Hammer), and each measurement included six measurements (Chart 2-10).

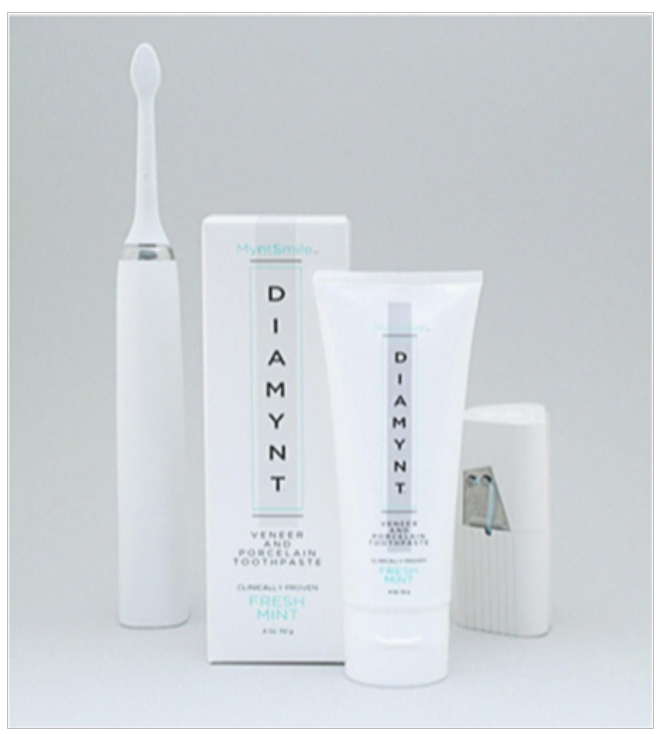

Chart 2

\section{Vita Profilometry Diamynt}

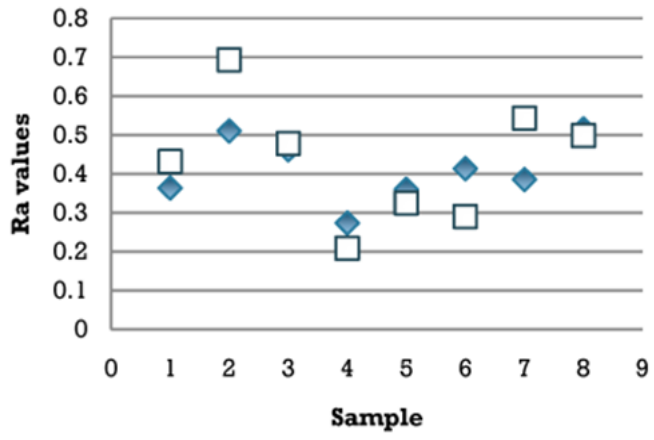

$\diamond$ Baseline $\square 10000$ cycles

\section{Chart 3}

\section{Conclusion}

Effects of toothbrush abrasion were shown to be material and toothpaste dependent. In Addition: All samples were measured with three readings on random areas within $5 \mathrm{~mm}$ of the center with a Konica-Minolta Spectrophotometer for $\mathrm{L}^{*} \mathrm{a} * \mathrm{~b} *$ values before and after brushing, however, data analysis has not been completed yet. Future directions for this project will be the continuation of measurement of surface roughness using an optical profilometer to increase representation of the sample through increasing total measurement area.

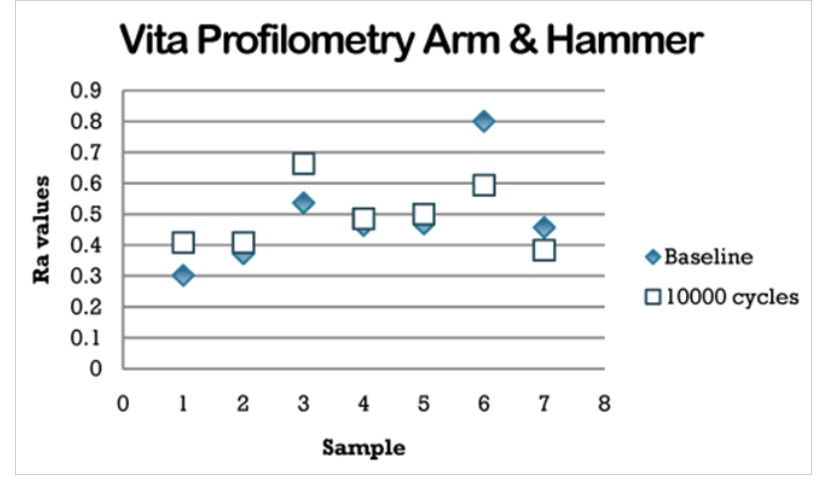

Chart 4

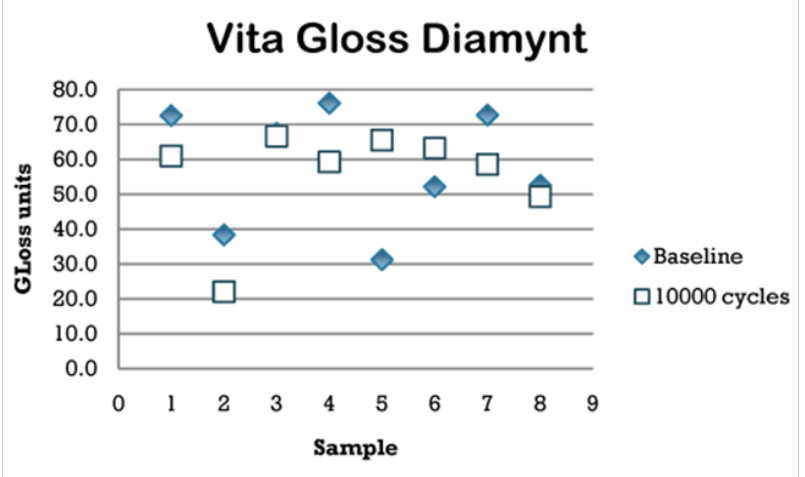

Chart 5

Vita Gloss Arm \& Hammer

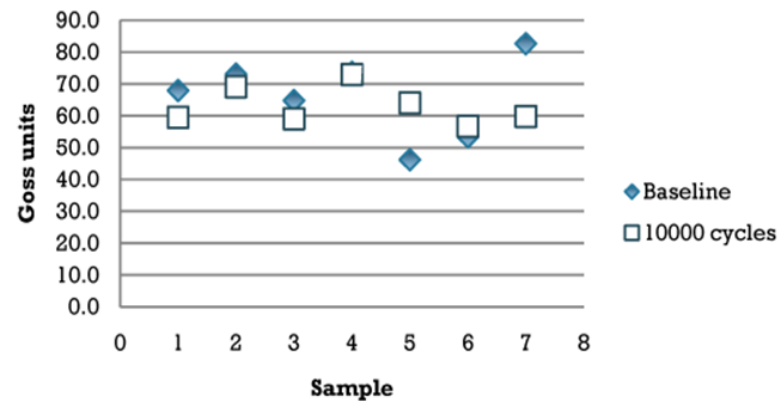

Chart 6

\section{Ceramco Profilometry Diamynt}

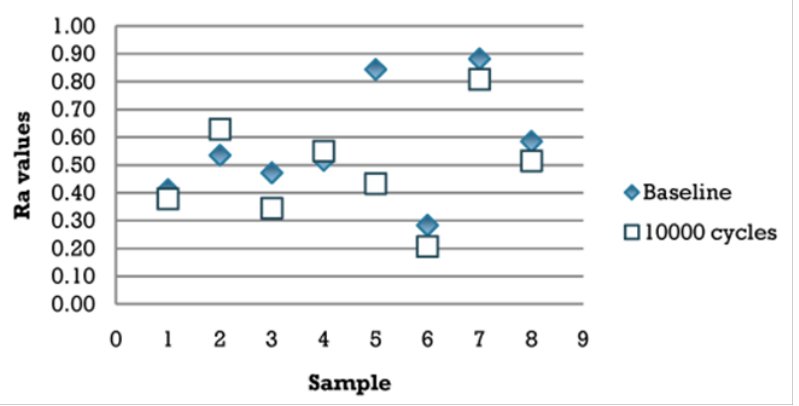

Chart 7 


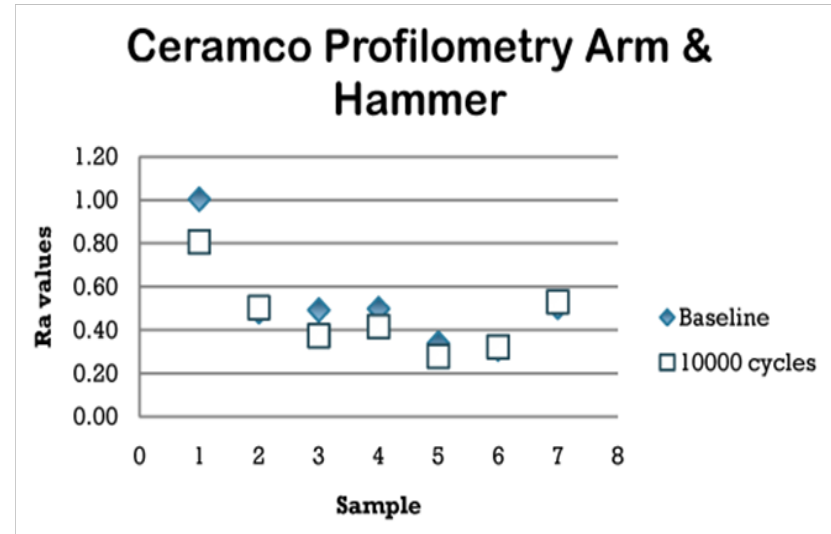

Chart 8

\section{Ceramco Gloss Arm \& Hammer}

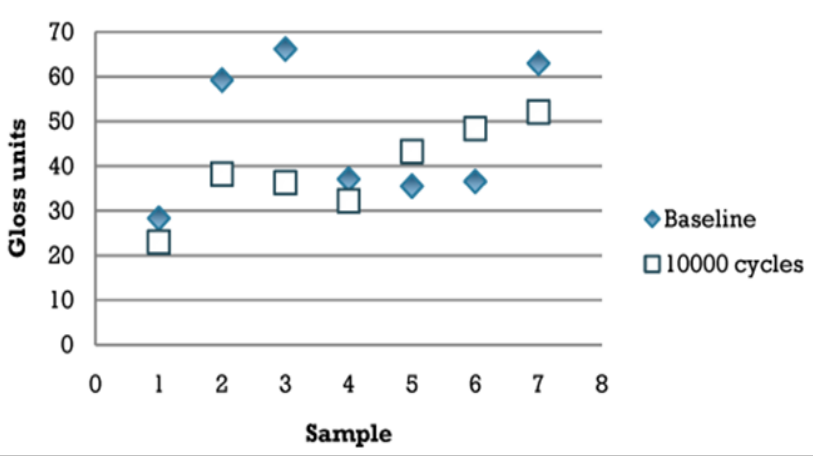

Chart 9

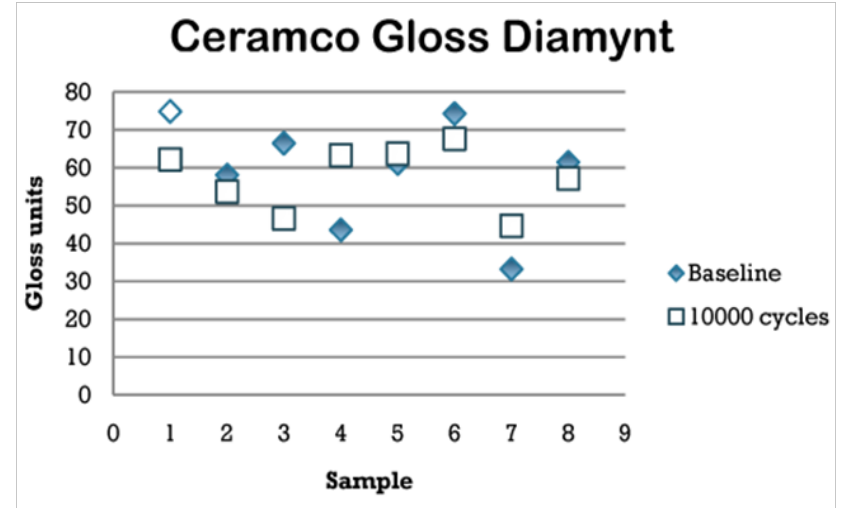

Chart 10

\section{Acknowledgments}

UTHSC College of Dentistry Alumni Endowment Fund and Tennessee Dental Association Foundation.

\section{Conflict of interest}

The author declares that there is no conflict of interest. 